\title{
Ameloblastoma em maxila: sugestão de abordagem cirúrgica
}

\author{
Maxillary ameloblastoma: suggestion of a surgical approach \\ Ameloblastoma en maxila: propuesta de abordaje quirúrgico \\ Thalles Moreira SUASSUNA ${ }^{1}$ \\ Júlio Leite de ARAÚJO-JUNIOR ${ }^{2}$ \\ Tácio Candeia LYRA ${ }^{2}$ \\ Joaquim Celestino da SILVA-NETO ${ }^{3}$ \\ José Wilson NOLETO \\ Eduardo DIAS-RIBEIRO \\ 'Mestrando em Odontologia da Universidade Federal da Paraíba (UFPB) 58051-900, João Pessoa - PB, Brasil \\ ${ }^{2}$ Residente de Cirurgia e Traumatologia Buco-Maxilo-Facial, Hospital Universitário Lauro Wanderley (UFPB) 58051-900, João Pessoa - PB, Brasil \\ ${ }^{3}$ Coordenador da Residência em Cirurgia e Traumatologia Buco-Maxilo-Facial do Hospital Getúlio Vargas 50630-060, Recife-PE, Brasil \\ ${ }^{4}$ Professor da Universidade Federal da Paraíba (UFPB) e da Residência em Cirurgia e Traumatologia Buco-Maxilo-Facial do Hospital Universitário \\ Lauro Wanderley (UFPB) 58051-900, João Pessoa - PB, Brasil
}

\section{Resumo}

Introdução: O ameloblastoma é um tumor benigno, localmente invasivo, originário do epitélio odontogênico e é a neoplasia odontogênica mais comum. Apresentam crescimento lento e sua ocorrência na maxila é pouco frequente. Objetivo: Discutir os métodos de tratamento para os ameloblastomas em maxila e demonstrar a utilidade da osteotomia Le Fort I na abordagem destas lesões. Material e Método: Estudo descritivo de relato de caso. Resultados: Observou-se a erradicação da lesão com uma abordagem de baixa morbidade e que permitiu bom resultado estético e funcional. Conclusão: A ressecção é o método mais indicado para tratamento dos ameloblastomas sólidos, e a sua realização utilizando a osteotomia Le Fort I podem trazer vantagens tanto no trans quanto no pós-operatório.

Descritores: Ameloblastoma; Maxila; Osteotomia de Le Fort.

\section{Abstract}

Introduction: Ameloblastoma is a benign, locally invasive tumor that originates from the odontogenic epithelium and is the most common odontogenic neoplasm. They present slow growth and its occurrence in the maxilla is infrequent. Objective: To discuss the treatment methods for maxillary ameloblastomas and to demonstrate the usefulness of Le Fort I Osteotomy in the approach to these lesions. Material and Method: Descriptive study of case report. Results: It was observed eradication of the lesion with a low morbidity approach and that allowed a good esthetic and functional result. Conclusion: Resection is the most appropriate method for the treatment of solid ameloblastomas, and its performance using the Le Fort I osteotomy can have advantages both in the trans and in the postoperative period.

Descriptors: Ameloblastoma; Maxilla; Osteotomy, Le Fort.

\section{Resumen}

Introducción: El ameloblastoma es un tumor benigno, localmente invasivo, originario del epitelio odontogénico y es la neoplasia odontogénica más común. Se presenta un crecimiento lento y su ocurrencia en la mandíbula es poco frecuente. Objetivo: Discutir los métodos de tratamiento para los ameloblastomas en maxila y demostrar la utilidad de la osteotomía Le Fort I en el abordaje de estas lesiones. Material Y Método: Estudio descriptivo de relato de caso. Resultados: Se observó la erradicación de la lesión con un abordaje de baja morbilidad y que permitió buen resultado estético y funcional. Conclusión: La resección es el método más indicado para el tratamiento de los ameloblastomas sólidos, y su realización utilizando la osteotomía Le Fort I pueden traer ventajas tanto en el trans como en el postoperatorio. Descriptores: Fracturas Óseas; Hueso Nasal; Traumatismos Faciales.

\section{INTRODUÇÃO}

O ameloblastoma é um tumor benigno, localmente invasivo, originário do epitélio odontogênico $^{1-3}$. É a neoplasia odontogênica mais comum. Podem surgir dos restos da lâmina dentária, de um órgão do esmalte, do epitélio de cistos odontogênicos ou das células basais da mucosa oral $^{1-3}$. Esta patologia não possui marcada predileção por raça ou gênero, e costuma acometer mais adultos jovens, entre a segunda e quarta décadas de vida ${ }^{1-4}$.

Pode se apresentar como sólido (multicístico), unicístico, periférico ou uma mistura desses padrões ${ }^{1-3}$. A variante sólida é a mais comum (86\% dos casos) e costuma ser mais agressiva. Apenas 10 a $20 \%$ dos ameloblastomas são encontrados na maxila e quando ocorrem costumam ser sólidos e se localizar na região de molares ${ }^{1,4}$.

A apresentação usual é de uma tumefação ou expansão dos ossos gnáticos, lenta e indolor, podendo comprometer o tecido mole adjacente $e^{1,5}$. Radiograficamente pode ser visto uma lesão radiolúcida uni ou multilocular, além de reabsorção radicular e deslocamento dental ${ }^{1,6}$.
O tratamento deste tumor deve ser através de remoção cirúrgica e para isso há vários métodos relatados que vão desde a marsupialização associada a curetagem até a ressecção em bloco com margem de segurança ${ }^{5,6}$. Este último método costuma ser o mais indicado por resultar em menores índices de recorrência ${ }^{4,7,8}$, no entanto a sua execução na região posterior de maxila pode pôr estruturas nobres em risco de injúrias e não obter precisão em conseguir margens cirúrgicas livres ${ }^{9,10}$.

Assim, o objetivo deste trabalho é, ilustrando com um caso clínico, propor e descrever uma abordagem que facilite a delimitação da lesão e minimize as dificuldades para remoção do ameloblastoma em maxila.

\section{CASO CLÍNICO}

Paciente melanoderma, do gênero masculino, 47 anos, foi encaminhado ao Ambulatório de Cirurgia Buco-Maxilo-Facial do Hospital Getúlio Vargas em Recife-PE com resultado de biópsia prévia apontando um ameloblastoma. O tempo de 
evolução relatado na anamnese foi de cerca de dois anos.

Clinicamente havia aumento de volume na região posterior do processo alveolar à esquerda, com consistência borrachoide à amolecida, coloração variando do vermelho eritematoso a áreas esbranquiçadas, telangiectasia em alguns pontos, não ulcerada, levemente sangrante à manipulação, indolor e com cerca de $3 \mathrm{~cm}$ de comprimento (Figura 1).

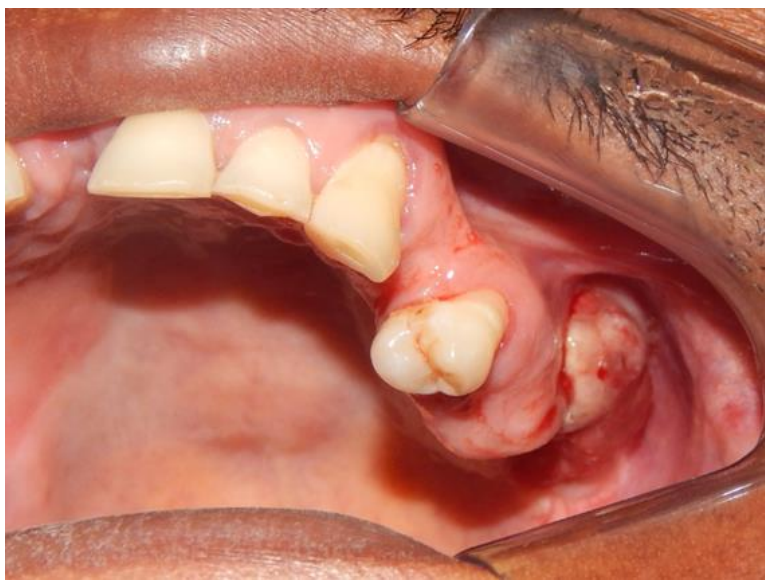

Figura 1: Aspecto clínico da lesão na região posterior da maxila à esquerda. Observe que o aumento de volume é discreto.

$\mathrm{Na}$ Tomografia Computadorizada era visível lesão isodensa, causando erosão óssea na parede vestibular da maxila esquerda, rechaçamento o seio maxilar e fazendo limite com o processo pterigóide e fossa nasal medialmente (Figura 2).

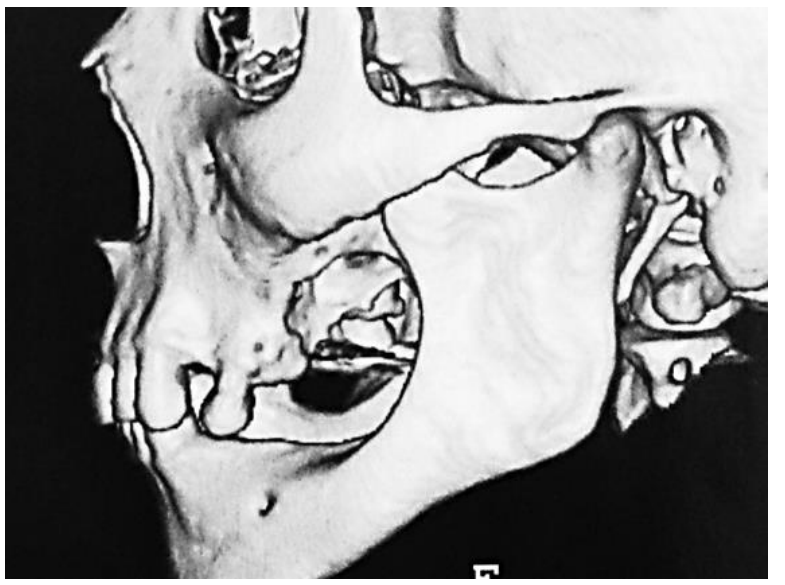

Figura 2: Reconstrução tomográfica tridimensional que evidencia lesão que erodiu a região de tuberosidade e processo pterigoide e crescia em direção póstero-superior.

Como tratamento planejou-se fazer um "down fracture" para melhorar o acesso à lesão e proceder com a ressecção. No pré-operatório foram feitos modelos de gesso dos arcos dentais e montagem dos mesmos em articulador, para confecção de um guia oclusal que permitisse fixar a maxila na posição original após a remoção do tumor.

O procedimento cirúrgico se deu através do acesso vestibular total de maxila, osteotomia Le Fort I (Figura 3) e abaixamento da maxila após soltar todas as resistências ósseas nos pilares. Após mobilizar a maxila, foi realizada a ligadura da artéria palatina descendente para prevenir um possível sangramento durante o procedimento. A tração da maxila para frente e para baixo, separando-a da base do crânio, permitiu bom acesso para a osteotomia, que foi realizada de forma semelhante a uma segmentação de maxila (Figura 4).

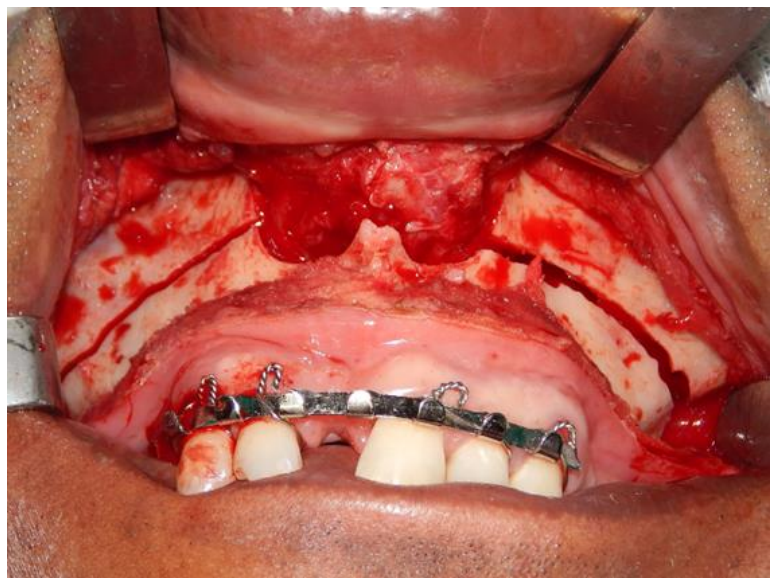

Figura 3: Acesso intra-oral circumvestibular e Osteotomia Le Fort I executada na maxila.

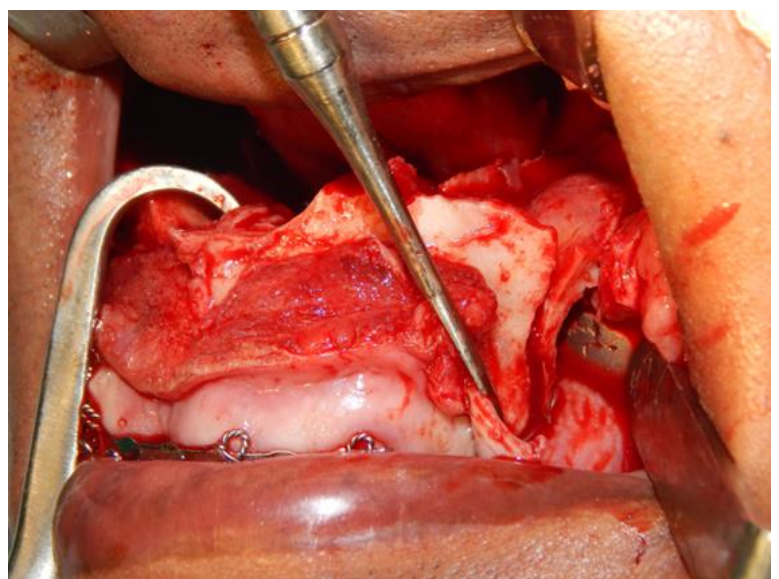

Figura 4: Maxila abaixada e após a osteotomia de segmentação. Observe que a mesma está posicionada para baixo e para frente o que facilitou a delimitação e remoção do tumor.

Após conclusão da osteotomia e a separação dos tecidos moles, a lesão foi totalmente removida (Figuras 5 e 6). Em seguida o restante do osso foi fixado na posição original (Figura 7).

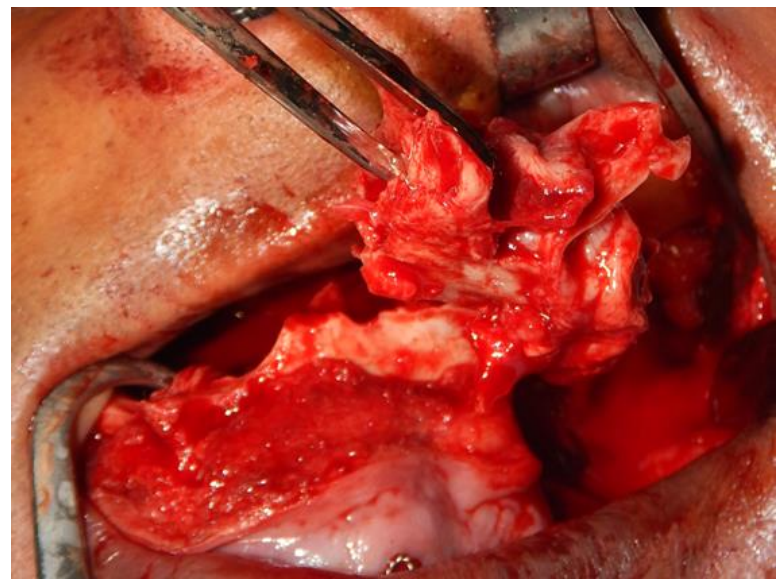

Figura 5: Remoção da porção óssea que continha o tumor. 


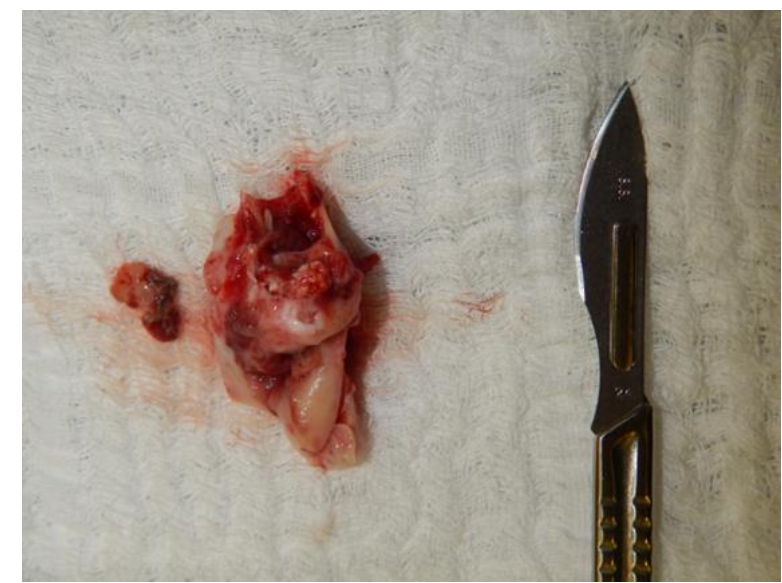

Figura 6: Tumor removido com margem de segurança de $1 \mathrm{~cm}$.

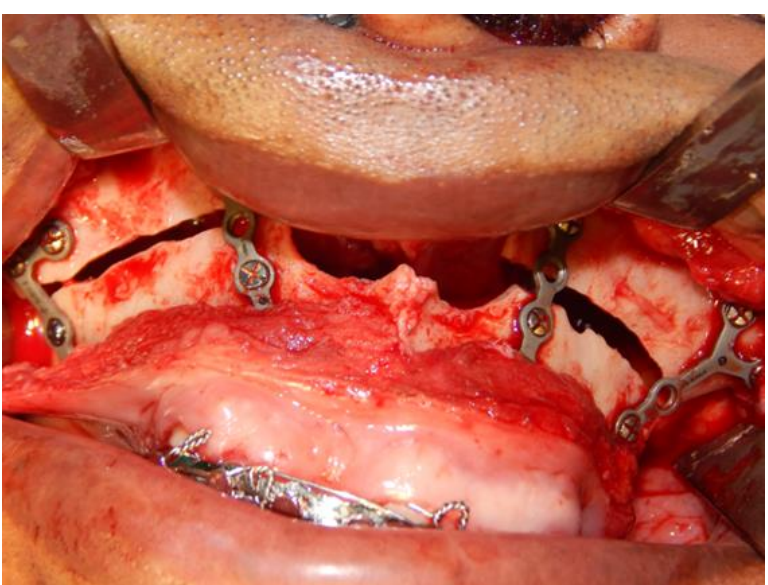

Figura 7: Maxila fixada na posição original, guiada por splint oclusal, após ressecção do ameloblastoma.

$\mathrm{O}$ exame anatomopatológico foi repetido e apontou um ameloblastoma plexiforme. O paciente evoluiu sem complicações, oclusão habitual e atualmente encontra-se em acompanhamento pósoperatório de cinco anos, sem exibir sinais de recidiva (Figura 8 ).

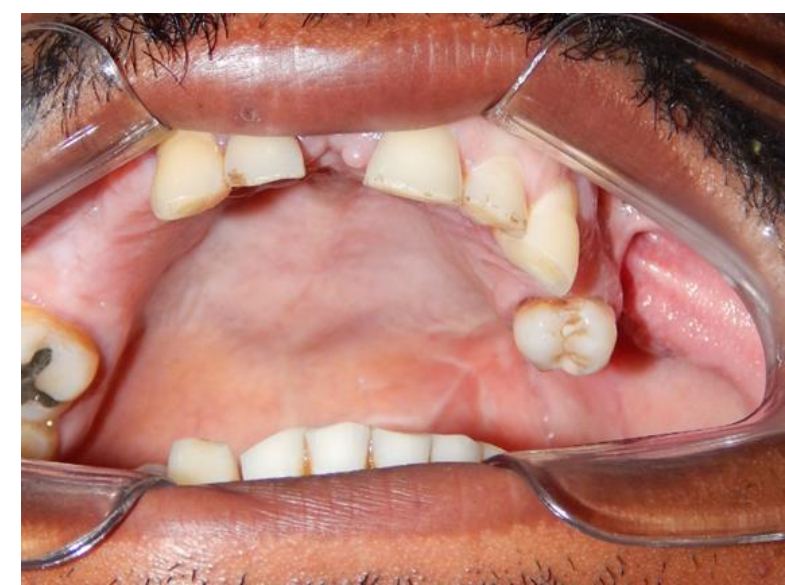

Figura 8: Aspecto clínico pós-operatório de 1 ano. Observe a boa cicatrização dos tecidos moles e ausência de comunicações buco-sinusais.

\section{DISCUSSÃO}

As formas de tratamento para os ameloblastomas são amplamente debatidas na literatura, no entanto ainda não há protocolo que se aplique a todos os casos e cada situação deve ser individualizada ${ }^{6,7}$. Critérios como a extensão e os limites do tumor, correlações anatômicas, variante histológica, idade e estado geral do paciente devem ser considerados para se escolher a melhor abordagem $^{11,12}$.

Pelo fato destas lesões se infiltrarem no osso radiograficamente intacto na periferia da lesão, o tratamento requer geralmente, intervenções radicais. Dessa maneira, a ressecção com margem de segurança é descrita com o método mais efetivo e tem sido o mais indicado por diminuir significativamente a chance de recorrência da lesão ${ }^{4,7,8}$.

Especialmente na maxila, que é um osso poroso e pneumático, a estrutura óssea não representa resistência ao crescimento destes tumores e isto é um fator que aumenta a agressividade e o potencial de infiltração do ameloblastoma ${ }^{10,13-15}$.

Pogrel et al. $^{6}$ indicam uma margem de segurança em torno de $10 \mathrm{~mm}$ ao redor dos ameloblastomas. No entanto Laborde et al. ${ }^{4}$ afirmam que, devido ao fato destas lesões serem mais agressivas na maxila, a margem de segurança deve ser ampliada.

Quando não é possível obter precisamente as margens de segurança, pode-se lançar mão de terapias complementares com através de solução de Carnoy ou com Nitrogênio líquido. No entanto, estes métodos são de difícil aplicação na maxila e possuem efeitos citotóxicos que prejudicam a cicatrização dos tecidos moles ${ }^{4}$.

A proximidade da maxila com o assoalho da órbita, fossa infratemporal e uma série de vasos e nervos nobres, faz com que os ameloblastomas na maxila sejam especialmente perigosos e potencialmente letais, pois possuem maior potencial de complicações e dificuldade de obtenção de margem cirúrgica adequada. Dessa forma, a escolha de uma abordagem que forneça bom acesso e visibilidade é essencial para o sucesso do tratamento ${ }^{11,12,14,15}$.

$\mathrm{Na}$ abordagem dos ameloblastomas sólidos de maxila vários acessos podem ser utilizados. Entre eles 0 acesso intra-oral vestibular maxilar (circumvestibular), o acesso de Weber-FergussonDiffembach, o "degloving" ("desenluvamento" do terço médio da face) $)^{10,16}$ e no presente artigo propomos o "down fracture" associado ao acesso intra-oral $^{17,18}$.

A osteotomia Le Fort I e o "down fracture" da maxila foi descrita primeiramente por Langenbeck em 1861 e popularizada pelos estudos de Bell em 1975. Atualmente essa técnica é amplamente utilizada para permitir o reposicionamento da maxila no tratamento de Deformidades Dento-faciais ${ }^{17}$. Ela consiste em, através de osteotomias com broca e/ou serra e cinzéis, simular uma fratura Le Fort I, que é horizontal e se estende da região de abertura pirifome 
à parede posterior da maxila bilateralmente ${ }^{10}$. Dessa maneira, toda a porção dento-alveolar e palatina da maxila, assoalho da fossa nasal, assoalho do seio maxilar e túber podem ser mobilizadas e separadas do restante do esqueleto fixo da face ${ }^{19}$.

O emprego desta técnica no acesso de patologias no terço médio já foi relatado na abordagem para tumores de maxila e de base de crânio $^{17-20}$, entretanto o seu emprego para tratamento de ameloblastomas não é difundido. Segundo os trabalhos iniciais que descreveram esta abordagem para os ameloblastomas, a principal vantagem dessa abordagem em relação à maxilectomia por via transalveolar é a excelente exposição tumoral, fato que permite visualização dos limites da lesão e um melhor acesso à artéria palatina descendente caso haja necessidade de sua ligadura ${ }^{17,18,20}$.

Como possíveis desvantagens dessa abordagem podem ser citadas o maior de tempo necessário para execução do procedimento, maior custo (devido à necessidade de material osteossíntese) e necessidade de um preciso planejamento para não haver alteração oclusal ao final da cirurgia ${ }^{17,20}$.

Deve ainda ser ressaltado que nem todos os ameloblastomas de maxila necessitarão dessa abordagem, especialmente aqueles muito pequenos, onde não há contato com a órbita ou com o pterigoide. Além disso, aqueles tumores de grandes dimensões poderão necessitar de abordagens mais invasivas como o acesso de "Webber-Fergusson", o "degloving" ou uma combinação de abordagens ${ }^{10,20}$.

\section{CONCLUSÃO}

As dificuldades em fazer uma abordagem para ressecção de lesões é uma dificuldade que pode ser minimizada por um planejamento cuidadoso e escolha correta da técnica. Neste contexto, a uso da osteotomia Le Fort I se apresentou como uma alternativa que possibilitou bom acesso para delimitação e remoção do tumor, com segurança e baixa morbidade.

\section{REFERÊNCIAS}

1. Kreppel M, Zöller J. Ameloblastoma - Clinical, radiological, and therapeutic findings. Oral Dis. 2018;24(1-2):63-6.

2. Taylor EM, Wu W, Kamali W, Ferraro P, Upton $\mathrm{N}$, Lin $\mathrm{J}$ et al. Medial femoral condyle flap reconstruction of a maxillary defect with a 3D printing template. J Reconstr Microsurg Open. 2017;2:e63-8.

3. Menezes LM, Souza CEL, Carneiro JT, Silva Kataoka MS, Júnior SDMA, Pinheiro, JDJV. Maxillary ameloblastoma in an elderly patient: report of a surgical approach. Hum Pathol. 2017;10:25-9.

4. Laborde A, Nicot R, Wojcik T, Ferri J, Raoul G.
Ameloblastoma of the jaws: Management and recurrence rate. Eur Ann Otorhinolaryngol Head Neck Dis. 2017;134(1):7-11.

5. Milman T, Ying GS, Pan W, LiVolsi V. Ameloblastoma: 25 year experience at a single institution. Head Neck Pathol. 2016;10(4):513-20.

6. Pogrel MA, Montes DM. Is there a role for enucleation in the management of ameloblastoma? Int $\mathrm{J}$ Oral Maxillofac Surg. 2009;38(8):807-12.

7. Antonoglou GN, Sándor GK. Recurrence rates of intraosseous ameloblastomas of the jaws: a systematic review of conservative versus aggressive treatment approaches and metaanalysis of non-randomized studies. J Craniomaxillofac Surg. 2015;43(1):149-57.

8. Almeida RA, Andrade ES, Barbalho JC, Vajgel A, Vasconcelos BC. Recurrence rate following treatment for primary multicystic ameloblastoma: systematic review and meta-analysis. Int $\mathrm{J}$ Oral Maxillofac Surg. 2016;45(3):359-67.

9. Rizzitelli A, Smoll N, Chae M, Rozen WM, Hunter-Smith DJ. Incidence and overall survival of malignant ameloblastoma. PLoS One. 2015;10(2): 0117789.

10.Nastri AL, Wiesenfeld D, Radden BG, Eveson J, Scully C. Maxillary ameloblastoma: a retrospective study of 13 cases. $\mathrm{Br} \mathrm{J}$ Oral Maxillofac Surg. 1995;33(1):28-32.

11.Guha A, Hart L, Polachova H, Chovanec M, Schalek P. Partial maxillectomy for ameloblastoma of the maxilla with infratemporal fossa involvement: A combined endoscopic endonasal and transoral approach. J Stomatol Oral Maxillofac Surg. 2018;119(3):212-15.

12. Quick-Weller J, Koch F, Dinc N, Lescher S, Baumgarten $\mathrm{P}$, Harter $\mathrm{P}$ et al. Intracranial ameloblastoma arising from the maxilla: an interdisciplinary surgical approach. J Neurol Surg A Cent Eur Neurosurg. 2017;78(5):582-87.

13.Abtahi MA, Zandi A, Razmjoo H, Ghaffari S, Abtahi SM, Jahanbani-Arkadani $\mathrm{H}$ et al. Orbital invasion of ameloblastoma: a systematic review apropos of a rare entity. J Curr Ophthalmol. 2018;30(1):23-34.

14.Bettoni J, Neiva C, Fanous A, Olivetto M, Demarteleire S, Demarteleire $\mathrm{C}$ et al. Brain ameloblastoma: metastasis or local extension report of a case and literature review. J Stomatol Oral Maxillofac Surg. 2018;119(5):436-39.

15. Yang R, Liu Z, Peng C, Cao W, Ji T. Maxillary ameloblastoma: factors associated with risk of recurrence. Head Neck. 2017;39(5):996-1000.

16.Kamalpathey LCK, Sahoo MGNK, Chattopadhyay CPK, Issar MY. Access Osteotomy in the Maxillofacial Skeleton. Ann Maxillofac Surg. 2017;7(1):98-103.

17. Alexander R, Weber WD, Theodos LV, Friedman 


\section{CONFLITO DE INTERESSES}

Os autores declaram não haver conflitos de interesse. tumors via Le Fort I downfracture: report of two cases and review of the literature. J Oral Maxillofac Surg. 1992;50(5):515-7.

18.Catunda IS, Melo AR, Medeiros Júnior R, Queiroz IV, Neto F, Leão JC. Osteotomia Le Fort I: Aspectos de interesse no tratamento de nasoangiofibroma juvenil. Rev cir traumatol bucomaxilo-fac. 2011;11(4):9-12.

19. Symington OG, Caminiti MF. Le Fort 1 down fracture approach for the treatment of a posterior maxillary ameloblastoma. J Can Dent Assoc. 1995;61(12):1048-52.

20.Iwaki LC, Tolentino ES, Lustosa RM, Jacomacci WP, Casaroto AR, Leite PC et al. Le Fort I osteotomy for the removal of a rare unicystic ameloblastoma lesion in the maxillary sinus. Gen Dent. 2016;64(3):16-9.
Submetido em 27/05/2018

Aceito em 27/06/2018 